\section{Size EfFect in Blunt Fracture: CONCRETE, ROCK, Metal}

By Zdeněk P. Bažant,' ${ }^{1}$ F. ASCE

Assmacr: The fracture front in concrete, as well as rock, is blunted by a zone of microcracking, and in ductile metals by a zone of yielding. This blunting causes deviations from the structural size effect known from linear elastic fracture mechanics (LEFM). The size effect is studied first for concrete or rock structures, using dimensional analysis and illustrative examples. Fracture is considered to be caused by propagation of a crack band that has a fixed width at its front relative to the aggregate size. The analysis rests on the hypothesis that the energy release caused by fracture depends on both the length and the area of the crack band. The size effect is shown to consist in a smooth transition from the strength criterion for small sizes to LEFM for large sizes, and the nominal stress $\sigma_{N}$ at failure is found to decline as $\left(1+\lambda / \lambda_{0}\right)^{-1 / 2}$ in which $\lambda_{0}=$
constant and $\lambda=$ relative structure size. This function is verified by Walsh's constant and $\lambda=$ relative structure size. This function is verified by Walsh's test data. If reinforcement is present at the fracture front and behaves elastically, the decline of $\sigma_{N}$ is of the same type but is shifted to larger sizes; howcome known size effects which have been attributed to rands ations within the structure should be explained by fracture mechanics, which gives a very different extrapolation to large structures. Finally, exploiting the five that in metals the size of the yielding zone at the fracture front is approximately constant it is shown by dimensional analysis that elastic-plastic fracture causes a similar size effect.

\section{INTAODUCTION}

In concrete structures as well as rock masses the fracture front is blunted by a zone of microcracking, whereas in ductile metals it is blunted by yielding. These front-blunting phenomena cause deviations from the structural size effect known from linear elastic fracture mechanics. Study of these deviations is the objective of this paper.

Understanding of the structural size effect is important for correct interpretation of test data. Most laboratory tests are carried out on a reduced scale, from which generalizations must be made for much larger real structures. With regard to the size effect, basically two types of theories may be distinguished:

1. Strength theory (or the concept of failure surfaces), in which the failure criterion is expressed in terms of stresses (or strains), calculated according to theories of elasticity, plasticity or viscoplasticity.

2. Linear elastic fracture mechanics, in which the failure criterion is expressed in terms of the energy consumed per unit crack length increment.

Although fracture mechanics might seem to be appropriate for con'Prof, of Civ. Engrg. and Dir., Center for Concrete and Geomaterials, Northwestern Univ. Evanston, Ill. 60201

Note--Discussion open until September 1, 1984. To extend the closing date one month, a written request must be filed with the ASCE Manager of Technical and Professional Publications. The manuscript for this paper was submitted for review and possible publication on May 3, 1983. This paper is part of the Joumal of Engineering Mechanics, Vol. 110, No. 4, April, 1984. OASCE, ISSN 0733-9399/ 84/0004-0518/\$01.00. Paper No. 18730 crete when the failure is due to cracking, the linear (classical) form of fracture mechanics was clearly shown to be inapplicable to concrete $(12,18$ $19,21-23,28-29,31,35-38$ ), except possibly for extremely large structures. For this reason, and perhaps also for reasons of simplicity, the present practice and building codes for concrete structures $(13,16,30,32,39)$ universally adhere to strength-based criteria. However, these criteria do not agree with fracture test data any better than classical fracture mechanics. Moreover, they are inherently inconsistent when applied in finite element analysis, since they lead to spurious dependence of the results on the element size $(4-7,11,17)$, whether or not a sudden stress drop or a gradual strain softening is considered. In fact, failure criteria in terms of stresses or strains are theoretically sound only in the sense of plasticity, which prohibits strain-softening, i.e. a decline of stress at increasing strain

Recently, however, it has been established $(4,11)$ that concrete does obey fracture mechanics provided that one uses a proper nonlinear form of fracture mechanics in which fracture front blunting by the large size of the microcracked zone is taken into account. The purpose of this paper is to examine the consequences of this finding for the structural size effect in various brittle failures of concrete structures, such as the diagonal shear failure of beams, slabs and panels, torsional failure of beams, punching shear failure of slabs or shells, cryptodome failure in the top slab of prestressed reactor vessels, some failures caused by bearing stresses, etc.

The size effect for rock fracture is similar to that for concrete, because of similarities of the fracture mechanism. In elastic-plastic metals, the mechanism of crack front blunting is different; nevertheless, it will be seen that the size effect is similar.

\section{Size Effects in Strength and Fracture Caiteria}

To separate the size effect from other influences, one must consider structures of different sizes but geometrically similar shapes (e.g., beams of the same span-to-depth ratio and the same crack length-to-depth ratio). According to standard criteria, failure is indicated by the condition $\sigma_{N}=f_{i}^{\prime}$ where $f_{i}^{\prime}=$ direct tensile strength of concrete, and $\sigma_{N}=$ nominal stress at failure characterizing the stress state at a certain critical point of the structure. For reasons of dimensionality, $\sigma_{N}=c_{N} P / b d$ where coefficient $c_{N}$ is independent of size and characterizes the structure shape and type of loading, $P=$ load or loading parameter, $d=$ characteristic dimension and $b=$ thickness. Thus, if one considers the plot of $\log \sigma_{N}$ versus $\log \lambda$ where $\lambda=d / d_{a}=$ nondimensional size parameter and $d_{a}$ = maximum size of aggregate, the locus of all failure states according to any kind of strength criterion is a horizontal line (Fig. 1), regardless of whether one uses elastic, plastic or viscoplastic analysis. The only difference between these types of analysis is the level at which the horizontal line is to be drawn. Fig. 1 shows examples for some elementary situations, such as beam bending, shear and torsion.

For linear elastic fracture mechanics, the plot of failure states is completely different. It is well known that $\sigma_{N}$ varies inversely as $\sqrt{d}$ for all linear fracture mechanics solutions $(14,15)$, and so the plot of $\log \sigma_{N}$ versus $\log \lambda$ is a straight line of slope $-1 / 2$; see Fig. 1 . 

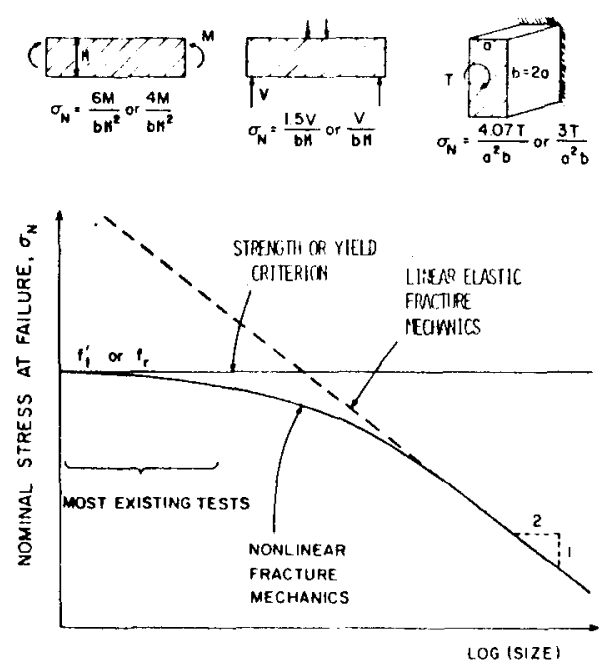

FIG. 1.-Size Effect According to Strength Criteria and Lineer or Nonlinear Fracture Mechanics

The true size effect in concrete structures represents a gradual transition from the horizontal line to the inclined straight line (Fig. 1), as we will see later.

\section{Blunt Crack Band Theory for Concrete or Rock}

In concrete, as well as mortars, rocks and certain ceramics, fracture is preceded by a gradual dispersed microcracking that occurs within a relatively large fracture process zone ahead of the tip of a continuous crack. As recently demonstrated $(2-7,11,17)$, fracture of this type may be modeled as the propagation of a band of uniformly and continuously distributed (smeared) cracks with a fixed width $w_{c}$ at the fracture front. At a certain distance behind the crack band front, the distributed cracks coalesce into one major crack; this need not be modeled, however, since only the situation at the fracture front matters for fracture propagation (Fig. 2).

It appears that the width $w_{c}$ of the crack band front must be treated as a material property, or else consistent numerical results independent of the method of analysis could not be obtained. Comparisons with numerous fracture test data confirm the constancy of $w_{c}$ and show that, if the results of stable direct tensile tests should be correctly represented at the same time, $w_{c}=n d_{a}$ where $d_{a}=$ maximum aggregate size (or grain size in rock), and $n=$ empirical constant, roughly $n=3$ for concrete $(4,11)$, and $n=5$ for rock $(2,9)$. The progressive microcracking in the fracture process zone may be described by a triaxial stress-strain relation $(4,11)$ that exhibits strain-softening with a gradual reduction of maximum principal stress $\sigma_{2}$ to 0 . The uniaxial special form of this stressstrain relation may be simply considered as a bilinear stress-strain dia-
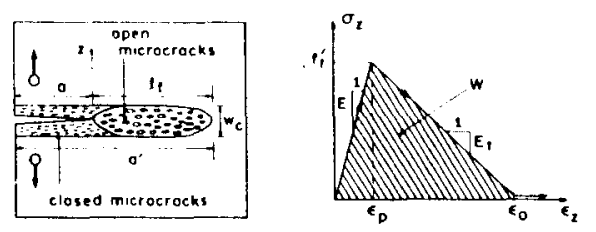

FIG. 2.-Crack Band Fracture and Ideallzed Tensile Stress-Strain Dlagram for Fracture Process Zone

gram, shown in Fig. 2, characterized by elastic modulus $E_{c}$, strength (peak stress) $f_{i}^{\prime}$, and strain-softening modulus $E_{t}$, which is negative. The energy consumed per unit advance of the crack band, called the fracture energy, may then be simply expressed $(4,11)$ as

$G_{f}=w_{c}\left(1-\frac{E_{c}}{E_{t}}\right) \frac{f_{t}^{\prime 2}}{2 E_{c}}$

By analyzing numerous test data, it was shown that $G_{\text {, may be predicted }}$ (with a coefficient of variation about $16 \%$ ) from the empirical formula $G$, $=0.0214\left(f_{i}^{\prime}+127\right) f_{i}^{\prime 2} d_{a} / E_{c}$ (with $E_{c}, f_{t}^{\prime}$ in pounds per square inch) (11). Substituting this formula into Eq. 1, $E_{\text {t }}$ can be solved as a function of $f_{i}, d_{a}, E_{c}$ and $w_{c}$. Note that $G_{f}$ is not the same as the apparent fracture energy determined on the basis of linear elastic fracture mechanics.

The experimental support for the crack band theory is broad. Not only does it agree with the fracture test data of various types and with direct tensile test data $(4,11)$, but also the strain-softening stress-strain relation used in this theory agrees with test data on short-time and long-time deflections of cracking beams (10) and gives the correct size effect for ductility (3).

\section{SOME llLustrative EXamples}

To illustrate the size effect resulting from the crack band theory, let us begin with some simple examples. Consider first a plain concrete center-cracked rectangular panel [Fig. 3(a)] of thickness $b$, width $2 d$, and a sufficiently large length $2 L$. The panel is loaded by vertical normal stresses $\sigma$ at top and bottom. The crack band is horizontal, symmetrically located, and has length $2 a$ and width $w_{c}=n d o$

We try to determine the value of $\sigma$ at which the crack band propagates. Before cracking, the strain energy density in the panel is uniform and equals $\sigma^{2} / 2 E_{1}$. The formation of the crack band may be imagined as an approximation, to relieve strain energy and stress from the "stress relief" area 1254361 in Fig. 3(a), in which the "stress diffusion" lines (27) $\overline{25}, \overline{45}, \overline{16}, \overline{36}$ have a certain fixed slope $k_{1}$ (close to 1 ). Thus, the total energy release from the panel is

$$
\begin{aligned}
& W=W_{1}+W_{2} \ldots \ldots \ldots \ldots \ldots \ldots \ldots \ldots \ldots \ldots \ldots \ldots \\
& \text { in which } W_{1}=2 k_{1} a^{2} b \frac{\sigma^{2}}{2 E_{c}}, \quad W_{2}=2 n d_{a} a b \frac{\sigma^{2}}{2 E_{c}}
\end{aligned}
$$




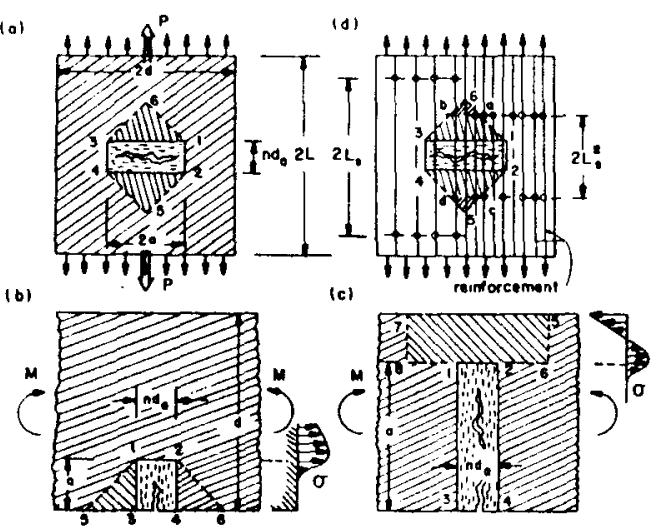

FIG. 3.-Examples of Crack Band Propagation

We imagine the top and bottom boundaries to be fixed during cracking, and so the contribution of the work of load $\sigma$ applied at the boundaries is zero. So, the potential energy release rate of the panel is $\partial \mathrm{W} / \partial a=$ $2\left(2 k_{1} a+n d_{a}\right) b \sigma^{2} / 2 E_{c}$. Due to the requirement of energy balance (first law of thermodynamics), $\partial W / \partial a$ must be equal to the energy consumed per unit crack band extension, i.e.

$\frac{\partial W}{\partial a}$

$\partial a$

After substituting Eq. 1 for $G_{f}$, we get an equation from which we may obtain the following solution:

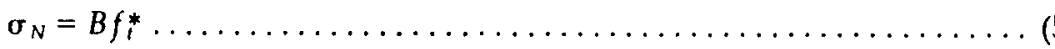

in which $\sigma_{N}=\sigma$ and

$f_{t}^{*}=\frac{f_{i}^{\prime}}{\sqrt{1+\frac{\lambda}{\lambda_{0}}}}$

and $B=\sqrt{1+\frac{E_{c}}{-E_{t}}} ; \quad \lambda_{0}=\frac{n}{2 k_{1}} \frac{d}{a}$.

in which $\lambda=d / d_{0}=$ relative structure size $(\lambda \geq n=3)$; and $B$ and $\lambda_{0}=$ constants when geometrically similar beams are considered. They are independent of the size. $f_{t}^{*}$ may be called the size reduced-strength. It is a characteristic of the entire structure, rather than of the material. Although the foregoing expressions for $B$ and $\lambda_{0}$ are only crude approximations, the form of Eq. 6 is exact, as we will show later.

As another example, consider a crack band of length $a$ and width $w_{c}$ $=n d_{a}(n \approx 3)$ in a rectangular unreinforced beam of thickness $b$ and depth $d$, subjected to bending moment $M$. First, consider that $a<<d$ (short cracks). In an approximate sense, the formation of the crack band may be imagined to relieve the strain energy from the "stress relief" area 1264351 in Fig. $3(b)$, in which the "stress diffusion" lines 15 and $\overline{26}$ have a certain empirical slope $k_{1}$ close to 1 . Before cracking, the strain energy density at the tensile face of beam is $\sigma_{1}^{2} / 2 E_{c}$ where $\sigma_{1}=6 \mathrm{M} / \mathrm{bd} d^{2}$, and the same value approximately applies over the whole region 1264351 if $a<<d$. Thus, the total energy release is $W=W_{1}+W_{2}, W_{1}=k_{1} a^{2} o_{1}^{2}$ $2 E_{c}, W_{2}=n d_{a} a \sigma_{1}^{2} / 2 E_{c}$, and the energy release rate of the beam, calculated at constant $M$, is $\partial W / \partial a=b\left(2 k_{1} a+n d_{a}\right)\left(6 M / b d^{2}\right)^{2} / 2 E_{c}$. Substituting this into Eq. 4, along with $G_{f}=n d_{a}\left(1-E / E_{t}\right) f_{t}^{\prime 2} / 2 E_{c}$ and $M$ $=\sigma_{N}(d-a)^{2} / c_{1}$ (where $c_{1}=$ const. $=6$ for elastic stress analysis, and $c_{1}=4$ for plastic stress analysis), we may express $\sigma_{N}$ from the resulting equation; this yields $\sigma_{N}=B f_{1}^{*}$ where $f_{1}^{*}$ is given again by Eq. 6 in which

$B=\frac{c_{1}}{6}\left(\frac{d}{d-a}\right)^{2} \sqrt{1+\frac{E_{c}}{-E_{1}}} ; \quad \lambda_{0}=\frac{n}{2 k_{1}} \frac{d}{a}$

Again $\lambda_{0}$ and $B$ are constants when geometrically similar beams are considered.

Finally, consider the same beam but $a-d<<d$ (short ligament) [Fig. $3(c)]$. Here we may write $\partial W / \partial a=(M \theta / 2)+W_{0}$ where $W_{0}=$ strain energy of the beam if no crack existed, which is independent of $a$, and $\theta=$ additional rotation caused by the crack band. Since the force resultants of the bending stresses over the ligament are zero, these stresses should affect only a region of size $d-a$, according to St. Venant's principle. Thus, we may imagine that the localized bending moment $M$ transmitted through the ligament $d-a$ affects only the region 1265781 in Fig. 3(c), with segments $\overline{18}$ and $\overline{26}$ equal to $k_{0}(d-a)$ where $k_{0}=$ empirical constant, close to 1 . Approximately, $\theta=\left[2 k_{0}(d-a)+n d_{a}\right]$ $M / E_{1} I_{1}$ where $I_{1}=b(d-a)^{3} / 12=$ inertia moment of the ligament section. Further, we may substitute $G$, according to Eq. 4 , and evaluate the derivative $\partial(M \theta / 2) / \partial a$ at constant $M$. Then we substitute this, as well as the relation $M=\sigma_{N}(d-a)^{2} / c_{1}$, into the condition $b G_{f}=\partial(M \theta / 2) /$ $\partial a(E q .4)$, and finally we express $\sigma_{N}$ from this condition; this yields again $\sigma_{N}=B f_{1}^{*}(\mathrm{Eq} .5)$ in which $f_{1}^{*}$ is again given by Eq. 6, although the constants are expressed differently:

$B=\frac{c_{1}}{6} \sqrt{1+\frac{E}{-E_{1}}} ; \quad \lambda_{0}=\frac{d}{d-a} \frac{n}{4 k_{0}}$

Eq. 6 can be similarly derived for various other situations, e.g., edgecracked panels, crack band in infinite medium, double-cantilever specimen, etc. The solutions are approximate in the evaluation of energy release; however, the approximate nature of the solution causes uncertainty only in the constants $k_{1}$ and $k_{0}$, but not in the form of Eq. 6. That form is exact.

\section{General Dimensional Analysis}

In the foregoing examples, $B$ and $C$ are independent of size, and the size effect is described by the same function (Eq. 6). This provokes sus- 
picion that Eq. 6 might be of general applicability. If so, it must be possible to verify it by dimensional analysis.

The geometry of a given structure may be characterized by some set of dimensions $d, l_{1}, l_{2}, \ldots l_{n}$, one of which represents the crack barid length, e.g., $l_{1}=a$. We consider all geometrically similar structures (with similar cracks) such that the ratios $\xi_{i}=l_{i} / d(i=1,2, \ldots n)$ are the same The size of the structure is characterized by a single characteristic dimension, $d$. As the preceding examples illustrate, the total release $W$ of strain energy of the structure consists, according to the crack band theory, of two parts $\left(W=W_{1}+W_{2}\right)$; one part $\left(W_{1}\right)$ is the energy released into the fracture front by the remaining (uncracked) part of the structure, and the second part $\left(W_{2}\right)$ represents the energy released from the material of the crack band. $W_{1}$ depends on the uncracked area (such as 136 and 245 in Fig. 3(a)) from which energy is released. This area is proportional to $a^{2}$, and is independent of $n d_{a}$. Therefore, $W_{1}$ is a function of $a$, and is independent of $d_{a}$. On the other hand, $W_{2}$ is proportional to the crack band area $\left(n d_{a}\right) a$. So, our fracture model rests on the following fundamental hypothesis:

Hypothesis I. - The total potential energy release $W$ caused by fracture is a function of both:

1. The length, $a$, of the fracture (crack band).

2. The area of the cracked zone, $n d_{a} a$.

It should be noted at this point that, in contrast to the preceding examples, we do not need to assume $W$ to be a sum of parts $W_{1}$ and $W_{2}$ (Eq. 2) corresponding to parts 1 and 2 of Hypothesis $1 . W$ can be a general function of $a$ and $n d_{a} a$.

The dependence of $W$ on length a characterizes the energy that is released into the fracture front from the surrounding uncracked structure [e.g., areas 136 and 245 in Fig. 3(a)], while the dependence of $W$ on $n d_{a} a$ characterizes the energy released from the cracking zone [e.g., zone 1243 in Fig. 3(a)]. As will be seen later, if $W$ depended only on a one would obtain linear fracture mechanics, and if it depended only on $n d_{a} a$ one would obtain the strength criterion.

Parameters $a$ and $n d_{\mathrm{a}} a$, however, are not nondimensional. They are allowed to appear only in a nondimensional form, which consists in the following nondimensional parameters:

$\alpha_{1}=\frac{a}{d} ; \quad \alpha_{2}=\frac{n d_{a} a}{d^{2}}$

representing the nondimensional crack band length and the nondimensional crack band area. Furthermore, $W$ must be proportional to the volume $d^{2} b$ of the structure and to the characteristic energy density $\sigma_{N}^{2} / 2 E_{c}$ where $\sigma_{N}=P / b d, P=$ given applied load or loading parameter, and $b$ $=$ thickness. In consequence of all these facts, the total strain energy release caused by cracking has the general form

$W=\frac{1}{2 E_{c}}\left(\frac{P}{b d}\right)^{2} b d^{2} f\left(\alpha_{1}, \alpha_{2}, \xi_{i}\right) \ldots$ in which $f=$ a certain continuous and continuously differentiable positive function, independent of size $d$. The precise definition of parameters $\xi_{1}$ and the precise dependence of $f$ on $\xi_{1}$ depends on the geometrical shape of the structure.

The condition for the crack band to propagate is $\partial W / \partial a=G_{f} b$ (Eq. 4). Thus, differentiating Eq. 11 at constant $\xi_{i}$ (similar structures), and noting that

$\frac{\partial f}{\partial a}=\frac{\partial f}{\partial \alpha_{1}} \frac{\partial \alpha_{1}}{\partial a}+\frac{\partial f}{\partial \alpha_{2}} \frac{\partial \alpha_{2}}{\partial a}$

we get $\left(\frac{f_{1}}{d}+\frac{f_{2} n d_{a}}{d^{2}}\right) \frac{p^{2}}{2 b E_{c}}=G$

in which we introduced the notations

$f_{1}=\frac{\partial f}{\partial \alpha_{1}} ; \quad f_{2}=\frac{\partial f}{\partial \alpha_{2}}$

Now, setting $P=\sigma_{N} b d, d=\lambda d_{a}$, and substituting Eq. 1 for $G_{f}$ (with $w_{c}$ $\left.=n d_{a}\right)$, we obtain

$\sigma_{N}=\frac{B f_{i}^{\prime}}{\sqrt{1+\frac{d}{\lambda_{0} d_{a}}}}$.

in which $B=\frac{1}{f_{2}} \sqrt{1-\frac{E_{c}}{E_{t}}} ; \quad \lambda_{0}=\frac{n f_{2}}{f_{1}}$

Thus, $\sigma_{N}=B f_{i}^{*}$ (Eq. 5) in which again $f_{i}^{*}=f_{i}^{\prime}\left(1+\lambda / \lambda_{0}\right)^{-1 / 2}$ (Eq. 6), with $\lambda=d / d_{a}$.

Eq. 6 or 15 , which is the basic result of this study, is thus verified in a general sense as a consequence of Hypothesis $I$. This equation expresses the selfsimilarity properties (1) of fracture of brittle heterogeneous materials.

Note that Eq. 6 or 15 is independent of the precise form of function $f\left(\alpha_{1}, \alpha_{2}, \xi_{i}\right)$. However, calculation of constants $B$ and $\lambda_{0}$ requires an accurate solution of this function. Often this is difficult to do. Calculations of $B$ and $\lambda_{0}$ in the previous examples are too crude, and it is preferable to determine $B$ and $\lambda_{0}$ by statistical regression of test data.

If the total energy release depended only on the length $a$ of fracture (first part of Hypothesis I), we would have $f_{2}=0$, and we would get $\sigma_{N}$ $=C / \sqrt{d}$ in which $C=\left(2 G_{f} E_{c} / f_{1}\right)^{1 / 2}=$ constant. This is the well-known size effect of linear fracture mechanics (Fig. 1).

If the total energy release consumed by fracture depended only on the area of the cracked zone (second part of Hypothesis I), we would have $f_{1}=0$, and we would get $\sigma_{N}=$ constant (Fig. 1). This is the type of size effect typical of plasticity, as well as all failure theories based on strength, critical strain, or failure surfaces in the stress or strain space. The present formulations of the so-called continuous damage mechanics also give the same type of size effect, i.e., $\sigma_{N}=$ constant.

For structures of a small size relative to the size of aggregate, i.e., for 
small $\lambda$, the value of $\lambda / \lambda_{0}$ in Eq. 6 may be neglected in comparison to 1 , and we then have $f_{i}^{*}=f_{i}^{\prime}$ and $\sigma_{N}=A f_{i}^{\prime}$. We see that the strength criterion (Fig. 1) is correct for small size structures. For structures of a very large size compared to the size of aggregate, 1 may be neglected in comparison to $\lambda / \lambda_{0}$ in Eq. 6 , and then

$f_{i}^{*} \simeq f_{i} \sqrt{\frac{\lambda_{0}}{\lambda}} \quad($ large $\lambda)$

We see that, for very large concrete structures, such as dams (or large rock masses), Eq. 6 asymptotically approaches the size effect of linear elastic fracture mechanics (Fig. 1). Obviously, Eq. 6 represents a gradual transition from the strength criterion for small structures to linear elastic fracture mechanics for large structures. For $\lambda>>\lambda_{0}$ the fracture energy dominates.

\section{Comparison with Test Data}

For the purpose of statistical regression analysis of test data, Eq. 6 may be transformed to a linear plot

in which $Y=\left(\frac{f_{i}}{\sigma_{N}}\right)^{2}, \quad \bar{a}=\frac{1}{B^{2}}, \quad \bar{b}=\frac{1}{B^{2} \lambda_{0}}$

Slope $\bar{b}$ and $Y$-intercept $\bar{a}$ may be easily determined by statistical regression, and then $B=1 / \sqrt{\bar{a}}, \lambda_{0}=\bar{a} / \bar{b}$.

The only extensive fracture test data in which geometrically similar specimens of different sizes were used appear to be those of Walsh (38). He tested three-point bent specimens of various depths, $d$, and the same ratio $a / d$ (for information on these tests, see Ref. 11 or 38 ). Walsh's test

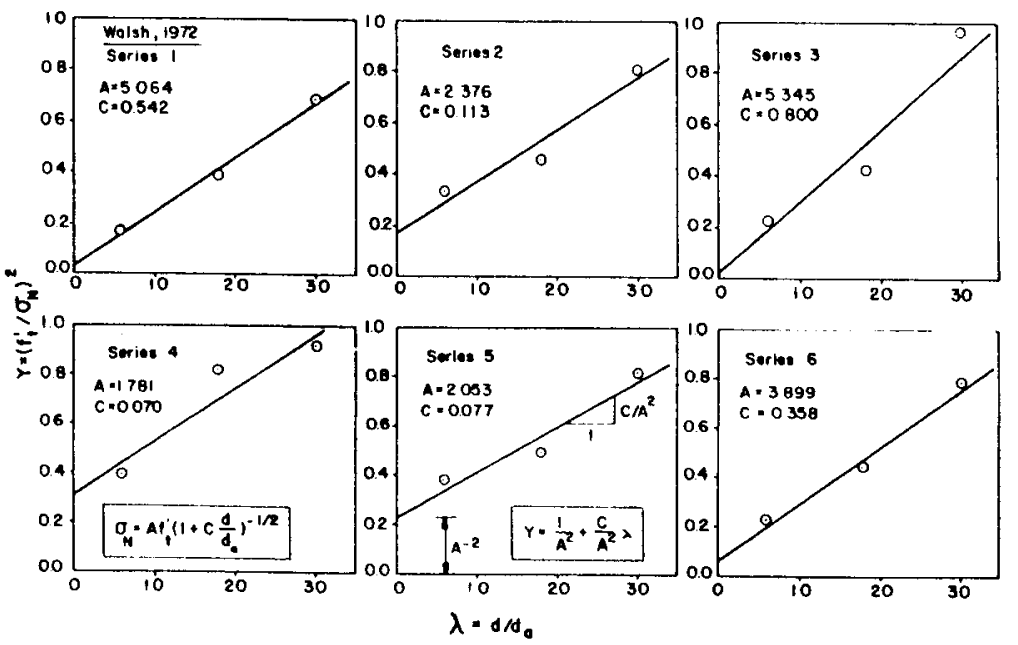

FIG. 4.-Regression Analysis of Fracture Test Data of Walsh (1972) results for six different concretes are plotted in Fig. 4 as $Y$ versus $\lambda$. If the test results followed the theory perfectly, these plots would have to be straight lines. Thus, the deviations of the data points from the regression lines shown in Fig. 4 represent the errors. From Fig. 4 we see that inclined straight lines agree with the basic trend of test data reasonably well. For the strength criterion, the regression lines would have to be horizontal, and for linear elastic fracture mechanics, the regression lines would have to pass through the origin $(\bar{a}=0)$. Obviously, none of this would agree with the tests (Fig. 4).

Eq. 6 may be also compared with laboratory test data for reduced scale structures. The largest set of test data exists in the literature with regard to the diagonal shear failure of reinforced beams without web reinforcement. The results of 296 beam tests from the literature, which have been statistically analyzed by J. K. Kim at Northwestern University (8), indicate again a good agreement with Eq. 6, much better than that for the strength criterion or for linear elastic fracture mechanics.

\section{Influence of Reinforcement and Bond Slip}

Reinforcement located in or near the fracture front may have influence, too. To examine it, consider the same center-cracked rectangular panel as before [Fig. $3(d)]$, reinforced by vertical steel bars which are spaced uniformly and so closely that a smeared modeling is possible. The panel is loaded on top and bottom by uniform normal stress $\sigma$.

Before cracking, the stress in concrete is $\sigma_{c}=\kappa_{c}=E_{c} /\left[E_{c}(1-p)+\right.$ $E_{s} p$ l; $p=$ steel ratio; and $E_{c}, E_{s}=$ Young's elastic moduli of concrete and steel. When cracks form, the steel bars slip against concrete over a certain length $2 L$, near the cracks, which may be estimated as $(6,17) L$ s $=\left(\sigma_{s} A_{b} / U_{b}^{\prime}\right)(1-p) /\left[(1-p)+p E_{s} / E_{c}\right]$, in which $p=$ steel ratio, $U_{b}^{\prime}=$ ultimate bond force per unit length of bar, as determined by pull-out tests, $A_{b}=$ cross section area of one steel bar, and $\sigma_{s}=$ steel stress at the cossing of crack band.

The presence of bond stresses along length $2 L_{s}$ complicates analysis. However, for the purpose of fracture analysis, the bond stresses are no very important, provided that the stiffness of the connection between the opposite sides of the crack band is modeled correctly. Thus, the frictional slip over length $2 L_{s}$ may be replaced by free (frictionless) slip ove a modified, free bond slip length $2 L_{s}^{*}$ (Fig. 3(d)). As an approximation, one may use $(6,17)$

$L_{s}^{*}=\frac{L_{s}}{2}=\frac{A_{b}}{2 U_{b}^{\prime}} \frac{1-p}{1-p+\frac{p E_{s}}{E_{c}}} \sigma$

Formation of fracture relieves the stress in concrete from the "stress relief" region 1254361 in Fig. 3 $(d)$, in which the "stress-diffusion" lines $16,25,36$, and 45 have a certain constant slope, $k_{1}$. The stress is, however, relieved to zero only if this region is entirely within the free bond slip length $2 L_{s}^{*}[$ Fig. $3(d)]$. If the crack band length, $a$, is so large that this region reaches beyond the free bond slip length, then the tensions in the steel bars introduce tensile stress into concrete within the triangular regions $6 a b$ and $5 c d$ in Fig. $3(d)$. The value of the stress in steel 
within the slip region, $\sigma_{s L}$, is less than (but probably close to) the stress that the steel carried before cracking, i.e., $\sigma_{s t}<\kappa_{s} \sigma$ where $\kappa_{s}=L_{s} /\left[E_{c}(1\right.$ $-p)+E_{s} p l$. Thus, the stress resultant per unit area, applied on these triangular regions, is less than $p \kappa_{s} \sigma$, which produces in concrete within the triangular regions a stress, $\sigma_{c}^{\prime}$, such that $\sigma_{c}^{\prime}<\kappa_{c}\left(p \kappa_{s} \sigma\right)$. So we may write

$\sigma_{c}=c_{\imath} p \kappa_{s} \kappa_{c} \sigma$

in which $c_{1}$ is a coefficient less, than 1 but probably close to 1 . The strain energy release from the panel of thickness $b$ may now be expressed as

$W=\left[\left(k_{1} a^{2}+a n d_{a}\right) \frac{\left(\kappa_{c} \sigma\right)^{2}}{2 E_{c}}-\frac{H_{a}}{k_{1}}\left(k_{1} a-2 L_{s}^{*}\right)^{2} \frac{\sigma_{c}^{\prime 2}}{2 E_{c}}\right] b$

in which $H_{a}=1$ if $k_{1} a>2 L_{s}^{*}$; and $H_{n}=0$ if $k_{1} a \leq 2 L_{s}^{*}$

In the energy balance, the energy consumed by bond slip should be also included. The maximum slip displacement of bars relative to concrete occurs at the crack band boundary and may be roughly estimated as $\left(f_{i}^{\prime} / E_{c}\right) L_{s}$. At the ends of length $2 L_{s}$, the slip is zero, and so the mean slip displacement is about $f_{i}^{\prime} L_{s} / 2 E_{c}$. The bond stress is roughly $U_{b}^{\prime}$ per unit length of bar, as determined from pull-out tests. The number of steel bars per unit cross section of panel is $p / A_{b}$ where $A_{b}=$ cross section area of one bar, and $L_{s}=2 L_{s}^{*}$. So the work of bond stresses over length $L_{s}$ per unit advance of the crack band is, approximately,

$W_{b}^{\prime}=\frac{p}{A_{b}} \frac{f_{i}^{\prime} L_{s}^{*}}{E_{c}}$

in which $b=$ panel thickness. In Eqs. 22 and 23 we may further substitute $L_{s}^{*}=L_{s} / 2=c_{L} A_{b} / 2 U_{b}^{\prime}$ where $c_{L}=\sigma_{s}-\sigma_{s}^{\prime}$

The energy balance condition for crack band advance may now be written as $b G_{f}+W_{b}^{\prime}=\partial W / \partial a$, where $G_{f}=n d_{a}\left(1-E_{c} / E_{t}\right) f_{t}^{\prime 2} / 2 E_{c}$. Differentiating Eq. 22 and substituting, we obtain $\sigma_{c}=B^{\prime} f_{i}^{*}$ where $\sigma_{c}=$ $\kappa_{r} \sigma$ and

$$
\begin{aligned}
& f_{i}^{*}=\frac{f_{i}^{\prime}}{\sqrt{1+\frac{\lambda}{\lambda_{1}}}} \\
& \lambda_{1}=\frac{B_{1}}{C_{1}} ; \quad B^{\prime}=\sqrt{\frac{A_{1}}{B_{1}}} ; \quad A_{1}=1+\frac{E_{c}}{-E_{t}}+\frac{p c_{L}}{n d_{a} f_{i}^{\prime}} ; \\
& B_{1}=1+H_{a} \frac{2 c_{L}}{n d_{a}} \frac{A_{b}}{U_{b}^{\prime}}\left(c_{t} p \kappa_{s}\right)^{2} ; \quad C_{1}=\frac{2 k_{1}}{n}\left[1-2 H_{a}\left(c_{t} p \kappa_{s}\right)^{2}\right] \frac{a}{d}
\end{aligned}
$$

Consider now geometrically similar panels (same $a / d$ ), with the same steel ratio, $p$, and bars of the same cross section, $A_{b}$, and the same steel stress at crack band crossing ( $c_{L} \simeq$ constant). Then, Eq. 24 indicates the same type of dependence on structure size parameter $\lambda$ as does Eq. 6 , except that $\lambda_{1}$ is larger than $\lambda_{0}$. This causes a shift of the asymptotic declining straight line in the plot of $\log f_{i}^{*}$ versus $\log \lambda$ to the right (see Fig. 5). Note also that if $A_{b}$ is increased with the structure size, the size
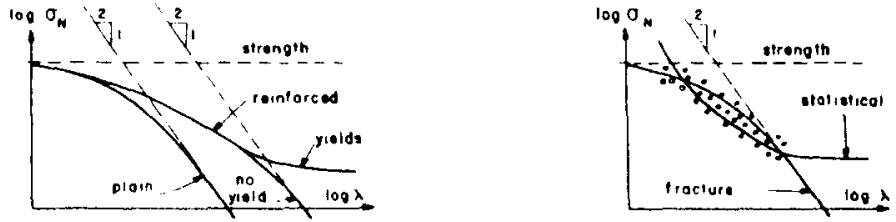

FIG. 5.-Size Effect when Reinforcement is Present at Fracture Front

FIG. 6-Comparison with Statistlcal Size Effect Due to Random Strength Distrlbution

effect becomes somewhat more pronounced since $f_{i}^{*}$ decreases as $A_{b}$ increases.

We thus see that the size effect in reinforced structures is less pronounced for smaller structures, but for large enough structures the size effect becomes just as significant as for unreinforced structures since the asymptotic slope remains $-1 / 2$. This is, however, true only if the reinforcement remains elastic.

For a long enough crack band, the opening in the center of its length becomes sufficiently large to cause the steel to yield, and that will completely alter the size effect. If the steel bars are yielding, the strains are so large that all tensile resistance of concrete is lost, and the load is then resisted by reinforcement alone. In that case the value of $\sigma$ becomes size independent. Therefore, in reinforced structures, the plot of $\log f_{t}^{*}$ versus $\log \lambda$ eventually stops decreasing and approaches a horizontal asymptote. However, this limiting plastic value might be too low for practical purposes. E.g., in diagonal shear of beams, it would mean designing the stirrups to carry the total shear force, rather than just a part of it.

\section{Further Considerations}

In laboratory testing, model structures have normally been made in the smallest size possible with regard to the aggregate size (cross sections of 5-15 aggregate diameters). Thus, the great majority of the laboratory tests of beams, plates, panels, slabs, shells, etc., thus far performed throughout the world are within the initial, nearly horizonta range of the diagram in Fig. 1. Obviously, such tests tell nothing about the size effect.

Present methods of design embodied in the codes for concrete structures are all based on strength criteria, elastic or plastic. This raises questions with regard to extrapolation to larger sizes typical of actual structures. The safety margin for a large structure might be smaller than thought, unless reinforcement yielding dominates. Therefore, it may be useful to reexamine the existing design procedures for those failures which are of brittle nature, e.g., the diagonal shear failure and torsion failure of beams, punching failure of slabs or shells, shear failure of deep beams and panels, cryptodome failure of a top slab in reactor vessels, etc. Recently it has become popular to analyze these failures according to the theory of plasticity, even though the failure may be caused by concrete 
cracking. This trend of research is questionable; if it has led to successful comparisons with test data, this may be simply because a wide range of structure sizes have not been tested in the laboratory.

The case of punching shear failure of slabs may serve as an illustration. Plasticity analysis can be made to agree with punching test results only if the tensile strength is considered to be about $f_{c}^{\prime} / 200$, which is about 20 times less than the correct value of tensile strength. The proper conclusion should not be that plasticity of concrete works, but that i does not work, and that fracture mechanics is, therefore, necessary. The small value of the nominal stress at failure is probably due to the fact that the existing laboratory test data do not pertain to the initial horizontal portion of the diagram in Fig. 1.

It should be kept in mind that many failures accompanied by cracking do not exhibit the fracture-type size effect. This is true of fracture-insensitive response, such as the bending failure of beams. One can decide whether this is the case by calculating the failure load twice; once for the actual tensile strength of concrete, and once for a zero tensile strength (a no-tension material). If both calculations yield about the same results, as is the case for bending of reinforced beams, then the designer does not need to worry about the problem analyzed here.

The results of fracture tests of concrete could no doubt be also successfully described by a theory in which one considers a line crack rather than a crack band and postulates at the crack tip a line segment characterized by a declining relation between the transverse normal stress and the crack opening displacement. This approach, initially introduced for metals by Knauss, Wnuk, Kfouri, Miller and Rice $(24,25,26,40)$, and pioneered for concrete by Hillerborg, Modéer and Petersson $(20,33)$, appears to be essentially equivalent to the crack band theory, with some minor exceptions $(4,11)$. Because of this equivalence, Eq. 6 should be applicable also to a fracture theory of this type.

\section{Question of Statistical Size Effect}

Because of the heterogeneity of concrete, its strength varies randomly throughout a concrete structure. This variation is independent of structure size. The stress gradient, on the other hand, normally varies inversely with the structure size, and so the region of nearly maximum stress (say, over $0.95 f_{i}^{\prime}$ ) becomes larger in a larger structure. Therefore, the chance of encountering low strength in the peak stress region is higher in a larger structure. The result is that the apparent strength necessarily declines with the structure size.

The decline stops, however, when the region of nearly maximum stress becomes much larger than the regions of low strength. Therefore, all theories of the statistical size effect produce a plot of $\log \sigma_{N}$ versus $\lambda$ which tends to a horizontal asymptote (Fig. 6). This is completely different from the fracture-mechanics size effect (Fig. 6), except when yielding of reinforcement dominates the response.

The statistical size effect has often been invoked to explain the apparent decrease of strength with structure size. On the basis of the present analysis, however, it appears that many observed size effects in concrete structures should be explained by fracture mechanics rather than by statistical variation of strength. The dependence of the apparent bending strength on the depth of plain concrete beams is an example (cf. Refs. 11, 33).

With regard to experimental confirmation, the importance of a sufficiently wide range of test data should be noted. If the test data do not cover a very large range of $\lambda$, the statistical size effect may seem to work (Fig. 6), even if fracture mechanics is the correct explanation. This may be obviously dangerous for extrapolations.

\section{Elastic-Plastic Faacture: Analogous Dimensional Analysis}

What is the size effect for elastic-plastic fracture mechanics of metals? It should be similar to Eq. 6, since elastic-plastic fracture can be described by models similar to Hillerborg's $(24-26,40)$, and Hillerborg's model is approximately equivalent to the crack band theory. In elastic-plastic fracture $(27,34)$, the crack tip is blunted by yielding, and a yielding zone, the size of which is essentially a material property, develops ahead of the crack tip. The far-away elastic stress-and-strain field is modified by the yielding zone and is approximately the same as in an elastic body containing a longer, equivalent crack. Let the length of the longer, equivalent (or fictitious) crack be $a+\Delta_{\text {p }}$ where $a$ is the actual crack length (Fig. 7). We may imagine that the longer crack relieves the stress from the triangular regions 567 and 568, while the crack of length $a$ in an elastic body would relieve the stress from the smaller regions 123 and 124 (Fig. 7). The area of these smaller regions is proportional to $a^{2}$, and so it depends only on $a$. Assuming that $\Delta_{p}<<a$, we find that the additional stress relief areas 132675 and 142685 are proportional to $a \Delta_{p}$, i.e., depend on $a \Delta_{p}$. A similar picture could be drawn for various types of fracture specimens. So it is reasonable to introduce the following hypothesis.

Hypothesis II.- The total potential energy release $W$ caused by fracture is a function of both:

1. The length, $a$, of the actual crack.

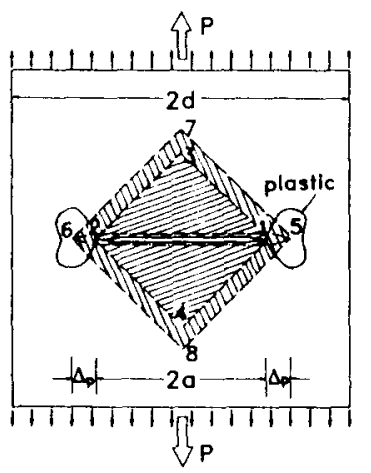

FIG. 7.-Effect of Ylelding Zone at Fracture Front in Metals 
2. The quantity $a \Delta_{p}$, where $\Delta_{p}$ is a constant having the dimension of length.

Let us now carry out a general dimensional analysis based on this hypothesis. Parameters $a$ and $a \Delta_{p}$ are not nondimensional. They must, however, appear in a nondimensional form, which is given by the following nondimensional parameters:

$\alpha_{1}=\frac{a}{d} ; \quad \alpha_{2}=\frac{a \Delta_{p}}{d^{2}}$

So, the total strain energy release caused by elastic-plastic fracture may be written as

$W=\frac{1}{2 E}\left(\frac{P}{b d}\right)^{2} b d^{2} f\left(\alpha_{1}, \alpha_{2}, \xi_{i}\right) \ldots$

in which $E=$ Young's elastic modulus. The crack propagates if $\partial W / \partial a$ $=\bar{G}_{f} b$ where $\bar{G}_{f}$ may be regarded as a material constant (effective fracture energy) representing the sum of the energy consumed (per unit crack extension) by the fracture process and of the energy consumed by plastic strain in the yielding zone at the crack front. Differentiating Eq. 27 at constant $\xi_{i}$ (geometrically similar structures), and using Eq. 12 with the notations in Eq. 14, we obtain $\left[\left(f_{1} / d\right)+\left(f_{2} \Delta_{p} / d^{2}\right)\right] P^{2} / 2 b E=\bar{G}_{f} b$. As a further important step we should note that, for the size of the yielding zone, Irwin showed that $\Delta_{p}=c_{p} \dot{G}_{f} E / \sigma_{y}^{2}(27)$ where $\sigma_{y}=$ the yield stress of the material and $c_{p}=$ some constant (of the order of one). Substituting this into the previфus relation, and setting $P=\sigma_{N} b d$, we may solve $\sigma_{N}$ and obtain

$\mathrm{\sigma}_{N}=\frac{B \sigma_{y}}{\sqrt{1+\frac{d}{d_{0}}}}$ with $d_{0}=\frac{f_{2}}{f_{1}} \Delta_{p}=k_{0} \frac{\bar{G}_{1} E}{\sigma_{y}^{2}} \ldots$

in which $k_{0}=c_{p} f_{2} / f_{1}$ and $B=2 /\left(c_{p} f_{2}\right)$. For geometrically similar structures, $k_{0}$ and $B$ are constants (nondimensional). Thus, $d_{0}$ is also a constant and may be called the characteristic size.

Due to the equivalence of Eq. 28 and Eq. 6 (or 15), a similar discussion could be repeated. In particular, Eq. 28 represents a gradual transition from the yield criterion for small structures $\left(d<<d_{0}\right)$ to linear elastic fracture mechanics for large structures $\left(d>>d_{0}\right)$.

\section{Conclusions}

1. Fracture of concrete structures is imagined to arise from propagation of a crack band whose width at the front is a fixed material property and equals about 3 times the maximum aggregate size. The size effect in brittle failure of concrete structures or rock masses may be analyzed on the basis of the hypothesis that the potential energy release caused by fracture depends on both: (a) The length of the crack band; and (b) the area of the crack band.

2. Dimensional analysis based on the foregoing basic hypothesis shows that, for structures that are geometrically similar (i.e., have the same shape), the nominal stress at failure varies with the structure size as (1 $\left.+\lambda / \lambda_{0}\right)^{-1 / 2}$ where $\lambda_{0}$ is a constant and $\lambda$ is the ratio of the size of the structure to the maximum size of the aggregate.

3. For the smallest structure that can be made with a given aggregate, the strength criterion governs, and for structures that are sufficiently larger, the energy criterion governs. The plot of the nominal stress at failure versus the size of the structure represents a smooth transition from the strength criterion to linear elastic fracture mechanics.

4. If reinforcement is present near the crack band front, and if it behaves elastically, the size dependence of the nominal stress at failure is of the same type but the validity of the strength criterion is extended and the transition to energy criterion occurs at a larger size. However, if the reinforcement yields, the decline of the nominal stress at failure with the structure size ceases.

5. Random variations of material strength give a different type of size effect. Various known size effects on strength, such as the dependence of the bending strength on the depth of a beam, should be explained principally by fracture mechanics rather than a statistical size effect.

6. The main physical mechanism that causes the size effect described here is not the microcracking but, more generally, fracture-front blunting of any type. Blunting due to plastic yielding, typical of metals, causes a similar type of size effect.

\section{ACKNOWLEDGMENT}

Partial financial support of U.S. Air Force Office of Scientific Research Grant No. 83-0009 to Northwestern University is gratefully acknowledged. Thanks are also due to B. H. Oh, Visiting Scholar at Northwestern University, for his help with numerical calculations for Fig. 4.

\section{ApPendix.-References}

1. Barenblatt, G. I., "Similarity, Self-Similarity and Intermediate Asymptotics," Consultants Bureau (Plenum Publ. Corp.) New York, N.Y., 1979 (transl. from Russian)

2. Bažant, Z. P. "Crack Band Model for Fracture of Geomaterials," Proc., 4th International Conference of Numerical Methods in Geomechanics, Z. Eisenstein, ed.. Edmonton, Alberta, May, 1982, Vol. 3, pp. 1137-1152.

3. Bažant, Z. P., "Instability, Ductility and Size Effect in Strain-Softening Concrete," Journal of the Engineering Mechanics Division, ASCE, Vol. 102, No. EM2, Apr., 1976, pp. 331-344.

4. Bažant, Z. P., "Mechanics of Fracture and Progressive Cracking in Concrete Structures," Report No. 83-2/428m, Center for Concrete and Geomaterials, Northwestern Univ., Evanston, Ill., Feb., 1983.

5. Bažant, Z. P., and Cedolin, L., "Blunt Crack Band Propagation in Finite Element Analysis." Journal of the Engineering Mechanics Division, ASCE, Vol 105 No. EM2, Proc. Paper 14529, Apr., 1979, pp. 297-315.

6. Bažant, Z. P., and Cedolin, L. "Fracture Mechanics of Reinforced Concrete," Journal of the Engineering Mechanics Division, ASCE, Vol. 106, No. EM6, Proc. Paper 15917, Dec., 1980, pp. 1287-1306; with Discussion and Closure Proc. Paper 15917, Dec, 1980, pp. 1287
in Vol. 108, EM2, 1982, pp. 464-471.

7. Bażant, Z. P., and Cedolin, L., "Finite Element Modeling of Crack Band Propagation," Joumal of Structural Engineering, ASCE, Vol. 109, No. ST2, Feb. 1983, pp. 69-92. 
8. Bažant, Z. P., and Kim, J. K., "Fracture Analysis of Diagonal Shear Failure of Concrete Beams," Report No. 83-5/4285. Center for Concrete and Geomaterials, Northwestern Univ., Evanston, III., May, 1983, (also American Concrete Institute Journal, in press).

9. Bazant, Z. P., and Oh, B. H., "Rock Fracture via Stress Strain Relations." Report No. 82-11/665r, Center for Concrete and Geomaterials, Northwestern Univ., Evanston, Ill., Nov., 1982.

10. Bažant, Z. P., and Oh, B. H., "Deformations of Cracked Reinforced Concrete Beams," American Concrete Institute Journal, Vol. 80, 1983.

11. Bažant, Z. P., and Oh, B. H., "Crack Band Theory for Fracture of Concrete," Materials and Structures, (RILEM, Paris), Vol. 16, 1983, pp. 155-177.

12. Brown, J. H., "Measuring the Fracture Toughness of Cement Paste and Mortar," Magazine of Concrete Research, Vol 24, No. 81, Dec., 1972, pp. 185-196.

13. "Building Code Requirements for Reinforced Concrete," ACI Standard 318377, American Concrete Institute, Detroit, Mich., 1977

14. Carpinteri, A., "Experimental Determination of Fracture Toughness Parameters $K_{i C}$ and $J_{K}$ for Aggregative Materials," Advances in Fracture Research (Proc., 5th International Conference on Fracture, Cannes, France, 1981) D. François, ed., Vol. 4, pp. 1491-1498.

15. Carpinteri, A., "Static and Energetic Fracture Parameters for Rocks and Concretes," Report, Istituto di Scienza delle Costruzion-Ingegneria, University of Bologna, Italy, 1980.

16. CEB-FIP Model Code for Concrete Structures, Comité Eurointernational du Béton CEB Bulletin No. 124/125-E, Paris, 1978.

17. Cedolin, L., and Bażant, Z. P., "Fracture Mechanics of Crack Bands in Concrete," Fracture Mechanic Methods for Ceramics, Rocks and Concrete, S. W. Freiman and E. P. Fuller, eds., Am. Soc. for Testing Materials STP745, 1981, pp. man and $221-236$.

18. Entov, V. M., and Yagust, V. I., "Experimental Investigation of Laws Governing Quasi-Static Development of Macrocracks in Concrete," Mechanics of Solids, (Translation from Russian), Vol. 10, No. 4, 1975, pp. 87-95.

19. Gjørv, O. E., Sørensen, S. 1., and Arnesen, A., "Notch Sensitivity and Fracture Toughness of Concrete," Cement and Concrete Research, Vol. 7, 1977, pp 333-344.

20. Hillerborg, A., Modéer, M., and Peterssson, P. E., "Analysis of Crack Formation and Crack Growth in Concrete by Means of Fracture Mechanics and Finite Elements," Cement and Concrete Research, Vol. 6, 1976, pp. 773-782.

21. Huang, C. M. J., "Finite Element and Experimental Studies of Stress Intensity Factors for Concrete Beams," thesis presented to Kansas State University, in 1981, in partial fulfillmẹnt of the requirements for the degree of Doctor of Philosophy

22. Kaplan, M. F., "Crack Propagation and the Fracture of Concrete," American Concrete Inst it ute Journal, Vol. .58, No. 11, Nov., 1961

23. Kesler, C. E., Naus, D. J., and Lott, J. L., "Fracture Mechanics-Its Applicability to Concrete," International Conference on the Mechanical Behavior of Materials, Kyoto, Aug., 1971.

24. Kfouri, A. P., and Miller, K. J., "Stress Displacement, Line Integral and Closure Energy Determinations of Crack Tip Stress Intensity Factors," Int. Journare Energy Determinations of Crack Tip Stress Intensity Factors,"
nal of Ves. and Piping, Vol. 2, No. 3, July, 1974, pp. 179-191.

25. Kfouri, A. P., and Rice, J. R., "Elastic/Plastic Separation Energy Rate for Crack Advance in Finite Growth Steps," in Fracture 1977, (Proceedings, 4th International Conference on Fracture), D. M. R. Taplin, ed., University of Waterloo Press, Vol. 1, 1977, pp. 43-59.

26. Knauss, W. C., "On the Steady Propagation of a Crack in a Viscoelastic Sheet; Experiments and Analysis," The Deformation in Fracture High Polymers, H. H. Kausch, ed., Plenum Press, 1974, pp. 501-541.

27. Knott, J. F., Fundamentals of Fracture Mechanics, Butterworths, London, England, 1973

28. Mindess, S., "The Application of Fracture Mechanics to Cement and Con- crete: A Historical Review," Chapter in State-of-the-Art Report of RILEM Technical Committee 50-FMD. (chaired by F.H. Wittmann), to be published

29. Mindess S Lawrence, F. V., and Kesler, C. E., "The J-Integral As a Fracture Criterion for Fiber Reinforced Concrete" Cement and Concrete Research, Vol. 7, 1977, pp. 731-742.

30. Naman. A F Prestressed Concrete Analysis and Design, McGraw Hill, New York, N.Y., 1982.

31. Naus, D. J., "Applicability of Linear-Elastic Fracture Mechanics to Portland Cement Concretes," thesis presented to the University of Illinois at UrbanaChampaign, Ill., in 1971, in partial fulfillment of the requirements for the degree of Doctor of Philosophy.

32. Nilson, A. H., Design of Prestressed Concrete, John Wiley \& Sons, Inc., New York, N.Y., 1978

33. Petersson, P. E. "Crack Growth and Development of Fracture Zones in Plain Concrete and Similar Materials," thesis presented to the Lund Institute of Technology, at Lund, Sweden, in 1981, in partial fulfillment of the requireTechnology, at Lund, Sweden, in 1981, in partial
ments for the degree of Doctor of Philosophy.

34 . posium at ASME Winter Annual Meeting, New York, ASCE AMD, Vol. 19, 1976, pp. 23-53.

35. Shah, S. P., and McGarry, F. J., "Griffith Fracture Criterion and Concrete," fournal of the Enginecring Mechanics Division, ASCE, Vol. 97, No. EM6, Proc. Paper 8597, Dec., 1971, pp. 163-1676.

36. Sok, C., Baron, J., and François, D., "Mécanique de la Rupture Appliquée au Béton Hydraulique," Cement and Concrete Research, Vol. 9.

37. Swartz, S. E., Hu, K. K., Fartash, M., and Huang, C. M. J., "Stress Intensity Factors for Plain Concrete in Bending-Prenotched Versus Precracked Beams," Report Department of Civil Engineering, Kansas State Univ., Kansas, 1981.

38 Walsh, P. "Fracture of Plain Concrete," The Indian Concrete Journal, Vol. 46, No. 11, Nov., 1979, pp. 469-470, and 476.

39. Winter, G., and Nislon, A. H., Design of Concrete Structures, 9th ed., McGraw Hill, New York, N.Y., 1979

40. Wnuk, M. P., "Quasi-Static Extension of a Tensile Crack Contained in Vis coelastic Plastic Solid," Iournal of Applied Mechanics, ASME, Vol. 41, 1974, No. 1. pp. $234-248$ 